University of Texas at El Paso

ScholarWorks@UTEP

$11-2015$

\title{
Science Is Helpful for Engineering Applications: A Theoretical Explanation of an Empirical Observation
}

Olga Kosheleva

The University of Texas at El Paso, olgak@utep.edu

Vladik Kreinovich

The University of Texas at El Paso, vladik@utep.edu

Follow this and additional works at: https://scholarworks.utep.edu/cs_techrep

Part of the Engineering Education Commons, and the Mathematics Commons

Comments:

Technical Report: UTEP-CS-15-88

To appear in Journal of Innovative Technology and Education

\section{Recommended Citation}

Kosheleva, Olga and Kreinovich, Vladik, "Science Is Helpful for Engineering Applications: A Theoretical Explanation of an Empirical Observation" (2015). Departmental Technical Reports (CS). 942.

https://scholarworks.utep.edu/cs_techrep/942

This Article is brought to you for free and open access by the Computer Science at ScholarWorks@UTEP. It has been accepted for inclusion in Departmental Technical Reports (CS) by an authorized administrator of ScholarWorks@UTEP.For more information, please contact Iweber@utep.edu. 


\title{
Science Is Helpful for Engineering Applications: A Theoretical Explanation of an Empirical Observation
}

\author{
Olga Kosheleva and Vladik Kreinovich \\ University of Texas at El Paso \\ 500 W. University \\ El Paso, TX 79968, USA \\ olgak@utep.edu,vladik@utep.edu
}

\begin{abstract}
Empirical evidence shows that when engineering design uses scientific analysis, we usually get a much better performance that for the system designed by using a trial-and-error engineering approach. In this paper, we provide a quantitative explanation for this empirical observation.
\end{abstract}

\section{An Empirical Observation}

Observation. At a recent annual conference of the American Society of Mechanical Engineers, one of the plenary talks was given by Dr. Hai Wang from Stanford University [4]. In his talk, Dr. Wang emphasized that scientific research is important for engineering practice. His argument was that without such a research, engineers tend to concentrate on one or few parameters of the system, and experience shows that much better performance can be achieved in a scientific approach, when all the parameters of the science can changed.

A similar idea was emphasized by Dr. Delbert Tesar from the University of Texas at Austin in his plenary talk at the same conference [3]. Specifically, Dr. Tesar emphasized that engineers tend to follow one - most "fashionable" direction, i.e., modify the values of only one (or few) parameters of the systems, and this severely limits their ability to reach the most efficient solutions to practical problems.

What we do in this paper. The purpose of this paper is to come up with a quantitative explanation of why the traditional trial-and-error engineering approach indeed leads to a drastic decrease in efficiency - as compared to allvariables optimization corresponding to the scientific approach. 


\section{Our Explanation}

Mathematical model of the situation: a general description. Let $x_{1}, \ldots, x_{n}, \ldots, x_{N}$ be parameters of the design, and let $f\left(x_{1}, \ldots, x_{n}, \ldots\right)$ be the value of the efficiency corresponding to the design parameters $x_{1}, \ldots, x_{n}, \ldots$

Without losing generality, we can assume that the parameters are sorted by the degree of their influence on efficiency: the parameter $x_{1}$ is the most influential, the parameter $x_{2}$ is the second influential, etc.

The influence of each parameter $x_{i}$ on the efficiency $f\left(x_{1}, \ldots, x_{n}, \ldots\right)$ can be measured by the corresponding partial derivative $c_{i} \stackrel{\text { def }}{=} \frac{\partial f}{\partial x_{i}}$. In these terms, the above assumption takes the form

$$
c_{1} \geq c_{2} \geq \ldots \geq c_{n} \geq \ldots
$$

Let us now describe how the scientific approach and the trial-and-error engineering approach compare in this model.

Scientific approach: a formal description. In the scientific approach, we can modify all the parameters to maximize the efficiency. A natural maximization step involving all the parameters is the gradient ascent step, in which we replace each original value $x_{i}$ with a modified value $x_{i}+\lambda \cdot \frac{\partial f}{\partial x_{i}}=x_{i}+\lambda \cdot c_{i}$, for some appropriate step $\lambda$.

After this step, in a linear approximation, the value of the efficiency changes to $f\left(x_{1}, \ldots, x_{n}\right)$ to

$$
\begin{gathered}
f\left(x_{1}+\lambda \cdot c_{1}, \ldots, x_{n}+\lambda \cdot c_{n}, \ldots\right)=f\left(x_{1}, \ldots, x_{n}, \ldots\right)+\sum_{i=1}^{N} \frac{\partial f}{\partial x_{i}} \cdot\left(\lambda \cdot c_{i}\right)= \\
f\left(x_{1}, \ldots, x_{n}, \ldots\right)+\lambda \cdot \sum_{i=1}^{N} c_{i}^{2} .
\end{gathered}
$$

So, the corresponding increase in efficiency

$$
\Delta_{s} \stackrel{\text { def }}{=} f\left(x_{1}+\lambda \cdot c_{1}, \ldots, x_{n}+\lambda \cdot c_{n}, \ldots\right)-f\left(x_{1}, \ldots, x_{n}, \ldots\right)
$$

is equal to

$$
\Delta_{s}=\lambda \cdot \sum_{i=1}^{N} c_{i}^{2}
$$

A trial-and-error engineering approach: a formal description. In the trial-and-error engineering approach, we only modify one (or a few) most promising parameters. Let $k$ denote the number of the modified parameters; then $k \ll N$ 
In this case, we only experiment with these $k$ parameters, so we only change these $k$ parameters. As a result, we change the values $x_{1}, \ldots, x_{i}, \ldots, x_{k}$ to the values $x_{i}+\lambda \cdot c_{i}$, while leaving all the values $x_{k+1}, \ldots, x_{n}, \ldots, x_{N}$ intact.

After this step, in a linear approximation, the value of the efficiency changes to $f\left(x_{1}, \ldots, x_{n}\right)$ to

$$
\begin{gathered}
f\left(x_{1}+\lambda \cdot c_{1}, \ldots, x_{k}+\lambda \cdot c_{n}, x_{k+1}, \ldots, x_{N}\right)= \\
f\left(x_{1}, \ldots, x_{k}, \ldots, x_{N}\right)+\sum_{i=1}^{k} \frac{\partial f}{\partial x_{i}} \cdot\left(\lambda \cdot c_{i}\right)= \\
f\left(x_{1}, \ldots, x_{n}, \ldots\right)+\lambda \cdot \sum_{i=1}^{k} c_{i}^{2}
\end{gathered}
$$

So, the corresponding increase in efficiency

$$
\Delta_{e} \stackrel{\text { def }}{=} f\left(x_{1}+\lambda \cdot c_{1}, \ldots, x_{k}+\lambda \cdot c_{k}, x_{k+1}, \ldots, x_{N}\right)-f\left(x_{1}, \ldots, x_{k}, \ldots, x_{N}\right)
$$

is equal to

$$
\Delta_{e}=\lambda \cdot \sum_{i=1}^{k} c_{i}^{2}
$$

To compare $\Delta_{s}$ and $\Delta_{e}$, let us use Zipf's law. We want to compare the values (1) and (2). The difference between these values depends on the values $c_{i}$.

It is known that in many practical situations, the decrease of the sorted values $c_{i}$ with the index $i$ is described by Zipf's law $c_{i} \approx c \cdot \frac{1}{i}$ for some constant $c$; see, e.g., $[1,2]$.

For this distribution, the sum (1) corresponding to large $N$ can be approximately described as an infinite sum

$$
\Delta_{s} \approx \lambda \cdot \sum_{i=1}^{\infty} c_{i}^{2}=\lambda \cdot c^{2} \cdot \sum_{i=1}^{\infty} \frac{1}{i^{2}} .
$$

It is known that the sum $\sum_{i=1}^{\infty} \frac{1}{i^{2}}$ is equal to $\frac{\pi^{2}}{6} \approx 1.64$ (for reader's convenience, the derivation of this formula is given in the Appendix). Thus, we have

$$
\Delta_{s}=\frac{\pi^{2}}{6} \cdot \lambda \cdot c^{2} \approx 1.64 \cdot \lambda \cdot c^{2} .
$$

In contrast, for the engineering trial-and-error approach, we similarly get

$$
\Delta_{e}=\left(1+\ldots+\frac{1}{k^{2}}\right) \cdot \lambda \cdot c^{2} .
$$


Resulting comparison. When the engineering approach is applied to only one parameter $k=1$, then we get

$$
\Delta_{e}=\lambda \cdot c^{2},
$$

which is only approximately $61 \%$ of the scientific increase (3).

For $k=2$, we get

$$
\Delta_{e}=\left(1+\frac{1}{4}\right) \cdot \lambda \cdot c^{2}=1.25 \cdot \lambda \cdot c,
$$

which is approximately $78 \%$ of the optimal improvement (3).

For $k=3$, we get

$$
\Delta_{e}=\left(1+\frac{1}{4}+\frac{1}{9}\right) \cdot \lambda \cdot c^{2} \approx 1.36 \cdot \lambda \cdot c,
$$

which is approximately $83 \%$ of the optimal improvement (3).

For $k=4$, we get

$$
\Delta_{e}=\left(1+\frac{1}{4}+\frac{1}{9}+\frac{1}{16}\right) \cdot \lambda \cdot c^{2} \approx 1.42 \cdot \lambda \cdot c,
$$

which is approximately $89 \%$ of the optimal improvement (3).

In all these cases, the use of the scientific approach indeed leads to significantly better increase in performance - more than $50 \%$ better for the typical case when only one most influential parameter is considered. Thus, a quantitative analysis indeed confirms the recommendation presented in [3] and [4]: it is important to use scientific results in engineering design.

\section{Acknowledgments}

This work was supported in part by the National Science Foundation grants HRD-0734825 and HRD-1242122 (Cyber-ShARE Center of Excellence) and DUE-0926721.

The authors are greatly thankful to Delbert Tesar for valuable discussions.

\section{References}

[1] A. I. Saichev, Y. Malevergne, and D. Sornette, Theory of Zipf's Law and Beyond, Springer Verlag, Berlin, Heidelberg, 2010.

[2] T. Tao, Solving Mathematical Problems: A Personal Perspective, Oxford University Press, Oxford, UK, 2006. 
[3] D. Tesar, "The future of Mechanical Engineering", Proceedings of the ASME 2015 International Mechanical Engineering Congress 83 Exposition IMECE'2015, Houston, Texas, November 13-19, 2015.

[4] H. Wang, "Combustion and future hybrid propulsion systems - opportunities and challenges", Proceedings of the ASME 2015 International Mechanical Engineering Congress \& Exposition IMECE'2015, Houston, Texas, November 13-19, 2015.

\section{A Appendix: Derivation of the Formula

$$
\sum_{i=1}^{\infty} \frac{1}{i^{2}}=\frac{\pi^{2}}{6}
$$

Main idea. It is known that in general, a polynomial

$$
P(x)=x^{n}+a_{1} \cdot x^{n-1}+\ldots+a_{n-1} \cdot x+a_{n}
$$

has $n$ roots (maybe complex) $x_{1}, \ldots, x_{n}$, and, in terms of these roots, this polynomial can be represented as

$$
P(x)=\prod_{i=1}^{n}\left(x-x_{i}\right) .
$$

In this equality, we assume that the coefficient at the largest degree is equal to 1 . If we divide each side of this equality by the product of all the values $x_{i}$, then we get a similar equality in which both sides have a coefficient at a free term (not depending on $x$ ) equal to 1 :

$$
Q(x) \stackrel{\text { def }}{=} \frac{P(x)}{a_{n}}=\prod_{i=1}^{n}\left(1-\frac{x}{x_{i}}\right),
$$

where

$$
Q(x)=1+b_{1} \cdot x+\ldots+b^{n} \cdot x_{n} .
$$

Here, the fact that the coefficient at a free is equal to 1 means that $Q(0)=1$.

Let us apply this idea to the sine function. The sine function $\sin (x)$ can be approximated by a polynomial with arbitrary accuracy: e.g., we can use more and more terms from the Taylor expansion

$$
\sin (x)=x-\frac{x^{3}}{3 !}+\frac{x^{5}}{5 !}-\ldots=x-\frac{x^{3}}{6}+\frac{x^{5}}{120}-\ldots
$$

For the sine function, $\sin (0)=0$, so we cannot directly apply the above idea to this function. However, if we divide $\sin (x)$ by $x$, then for $x \rightarrow 0$, the ratio has the limit 1 . So, we can apply the above formula and conclude that

$$
\frac{\sin (x)}{x}=\prod_{i}\left(1-\frac{x}{x_{i}}\right),
$$


where $x_{i}$ are all the roots of the ratio

$$
\frac{\sin (x)}{x}=1-\frac{x^{2}}{6}+\frac{x^{4}}{120}-\ldots
$$

It is known that $\sin (x)=0$ when $x=n \cdot \pi$ for an integer $n$. The root corresponding to $n=0$ is not the root of the ratio, so what is left are roots $\pm j \cdot \pi$ for $j=1,2, \ldots$ Substituting these roots into the above formula, we get

$$
\frac{\sin (x)}{x}=\prod_{j=1}^{\infty}\left(1-\frac{x}{j \cdot \pi}\right) \cdot\left(1-\frac{x}{j \cdot \pi}\right) .
$$

By using the formula $(1-a) \cdot(1+a)=1-a^{2}$, we can transform this product into

$$
\frac{\sin (x)}{x}=\prod_{j=1}^{\infty}\left(1-\frac{x^{2}}{j^{2} \cdot \pi^{2}}\right) .
$$

In general, $\left(1-a \cdot x^{2}\right) \cdot\left(1-b \cdot x^{2}\right)=1-(a+b) \cdot x^{2}+o\left(x^{2}\right)$, and, similarly,

$$
\left(1-a \cdot x^{2}\right) \cdot\left(1-b \cdot x^{2}\right) \cdot \ldots=1-(a+b+\ldots) \cdot x^{2}+o\left(x^{2}\right) .
$$

Thus, if we now expand both sides in Taylor series, we get

$$
1-\frac{x^{2}}{6}+\frac{x^{4}}{120}-\ldots=1-\sum_{j=1}^{\infty} \frac{1}{j^{2} \cdot \pi^{2}} \cdot x^{2}+\ldots
$$

By equating terms proportional to $x^{2}$ in both sides of this equality, we get

$$
-\frac{1}{6}=-\sum_{j=1}^{\infty} \frac{1}{j^{2} \cdot \pi^{2}}
$$

Multiplying both sides by $-\pi^{2}$, we get the desired formula

$$
\sum_{j=1}^{\infty} \frac{1}{j^{2}}=\frac{\pi^{2}}{6} .
$$

